\title{
Physiotherapists' and occupational therapists' perceptions of the assessment of stroke patients for musculoskeletal rehabilitation in the UK national health service
}

\author{
Christopher Golby ${ }^{1 \#}$, Gillian Lewando Hundt ${ }^{2}$, Vinesh Raja ${ }^{1}$ \\ ${ }^{1}$ Institute of Digital Healthcare, WMG, University of Warwick, Coventry, UK; *Corresponding Author: c.golby@warwick.ac.uk \\ ${ }^{2}$ Warwick Medical School University of Warwick, Coventry, UK
}

Received 18 April 2013; revised 20 May 2013; accepted 15 June 2013

Copyright (C) 2013 Christopher Golby et al. This is an open access article distributed under the Creative Commons Attribution License, which permits unrestricted use, distribution, and reproduction in any medium, provided the original work is properly cited.

\begin{abstract}
Background and Purpose: This exploratory study focused on the assessment of stroke patients for musculoskeletal rehabilitation in the United Kingdom National Health Service. It was the first phase of research on developing telerehabilitation for the assessment of patients who have had a stroke. The assessment of stroke patients for musculoskeletal rehabilitation is currently performed at the therapist's discretion using different outcome measures. When looking at the Department of Health's "National Clinical Guidelines for Stroke", it is stated that there are a variety of outcome measures, yet no particular one is recommended. It is specified that each rehabilitation sector should select its own methodologies. Aim: The aim of this exploratory study was to understand physiotherapists' and occupational therapists' perspectives of the strengths and weaknesses of current rehabilitative assessment of stroke patients in the UK National Health Service. Methods: This study had 2 parts, nonparticipant observation with 2 therapists and 3 patients, and 10 semi-structured interviews with 5 physiotherapists and 5 occupational therapists to identify current practice, problem areas, and what types of improvements could be made. Interviews were transcribed and analyzed using thematic coding. Results: Seven emergent themes were identified portraying how outcome measures are currently not being used in a standardized way within National Health Service
\end{abstract}

${ }^{*}$ Conflicts of interest: The authors of this paper would like to declare that there are no conflicts of interest. hospitals. This means that the feedback provided to patients, therapists and healthcare commissioners is limited. Therapists are currently performing more informal assessments each time a patient begins therapy and concerns are shown with these methods of assessment, including subjectivity, standardization issues and time. Interviewed therapists were clear that they believed that change is required within this field. Conclusions: This study raises concerns about the methodologies used for the assessment of stroke patients for rehabilitative purposes in the United Kingdom National Health Service.

Keywords: Stroke Rehabilitation; Musculoskeletal Assessment; Physiotherapy; Occupational Therapy

\section{INTRODUCTION}

There is an increasing incidence of stroke and stroke risk factors worldwide $[1,2]$. Those who survive a stroke require rehabilitation [3]. During rehabilitation, patients often require therapy for musculoskeletal problems such as reduced range of movement, balance and musculoskeletal pain, which is performed by physiotherapists (PTs) and occupational therapists (OT's) [4].

This study focused on the assessment of stroke patients who require musculoskeletal rehabilitation from stroke and the outcome measures used to evaluate their progress. The outcome measures that are used within the UK National Health Service (NHS) were evaluated.

Currently assessment of stroke patients in the NHS is performed at the discretion of therapists who choose and utilize different outcome measures. When looking at the UK Department of Health's National Clinical Guidelines 
for Stroke, this becomes clear:

"Measurement of function is central to rehabilitation. Many valid tools exist [patient outcome measures], and although these guidelines do not specify which ones should be used, some suggestions are made in the appropriate parts of the document. It is important that staff are trained in whichever scales are chosen to ensure consistency of their use within the team and an understanding of their limitations and purposes [4].”

Stroke rehabilitation in the United Kingdom (UK) can already be described as suffering from a multitude of problems such as the lack of time, staff, structure, cost and even knowledge [5-8]. In the area of assessment, problems become even more apparent. The way in which the NHS uses outcome measures has been criticized and new methods for assessing stroke patients for rehabilitative purposes that are "valid, reliable, responsive, and comprehensive" need to be developed $[9,10]$. It has also become apparent that there are no methods for assessment that can be applied to all circumstances [11]. It can even be stated that outcome measures are still not used effectively and routinely within musculoskeletal rehabilitation for stroke patients [12]. This research will involve a set of observational studies and interviews to evaluate what PTs and OTs in two NHS sites believe are the current strengths and weaknesses within this field and how they feel it could be improved.

\section{METHODOLOGY}

\subsection{Study Design}

Non-Participant observation was carried out in two clinical settings [13]. The researcher introduced himself to the therapist and patient at the beginning of a therapy session, took consent, and then observed; interacting as little as possible within the session. 2 therapists were observed at 3 sessions with 3 patients.

Subsequently, semi-Structured interviews [13] were carried out at two NHS stroke units in the Midlands, UK. These interviews used a topic guide to elicit the responses of 10 healthcare professionals, inclusive of 5 PTs and 5 OTs. The sample size aimed to capture some diversity of practice between professions and between therapists. Two Pilot interviews were performed and adjustments made to the topic guide. The results of these pilot interviews were not included in the study sample.

\subsection{Ethics}

Ethics permission was received from the UK National Research Ethics Service (NRES) after review by the Birmingham East, North and Solihull Research Ethics committee (REC). Information sheets and consent forms were issued to all healthcare staff. All interviewees were informed that participation was voluntary and all information would be kept confidential and anonymous.

\subsection{Recruitment}

Research and development departments within the NHS were asked to circulate emails to PTs and OTs asking whether they wished to be involved in an interview regarding the current methods of rehabilitative assessment for stroke patients in the NHS. PTs and OTs were then able to volunteer for the study by contacting the research team by email or phone. As shown in Table 1, the health professional participants were mainly female which reflects the predominant gender of these two professions. The three patients were male and were over 40 years of age recovering from strokes.

\subsection{Interviews}

The research team decided that an interview which was semi-structured and allowed respondents to control feedback in a thorough manner would elicit unbiased responses. This is the interview topic guide. The interviews were all undertaken by the same member of the research team (CG).

\subsection{Analysis}

During non-participant observation notes were taken. These notes are summarized and presented initially.

This was followed by interviews which were recorded and transcribed. Qualitative thematic analysis was used to evaluate them. In order to address inter-rater reliability, transcripts were read independently by two of the team (CG, GLH) with a thematic template being agreed upon after this. If required, sub-themes were created within a main theme. Figure 1 demonstrates the template that was established.

\section{RESULTS}

\subsection{Sample}

All 10 participants (5 PTs and 5 OTs) agreed to participate. These therapists are referred to as PT1-5 and OT1-5 in this analysis. Two of the therapists also agreed to partake in non-participant observation. PT1-5 and OT1-5 all worked within the Midlands Region, in the UK.

\subsection{Non-Participant Observation of Occupational Therapist with Patient 1}

Patient one had suffered a stroke in December 2009 and was observed in March 2011. They had made an

Table 1. Participant demographics.

\begin{tabular}{ccc}
\hline & Male & Female \\
\hline Physiotherapists & 1 & 4 \\
Occupational Therapists & 0 & 5 \\
Patients & 3 & 0
\end{tabular}


Table 2. Different outcome measures and times mentioned.

\begin{tabular}{cc}
\hline Outcome Measure & Times Mentioned \\
\hline Rivermead Mobility Index & 6 \\
Oxford Scale & 8 \\
Berg Balance Scale & 9 \\
Range of Movement/Goniometry & 8 \\
$10 \mathrm{~m}$ Walk & 5 \\
Ashworth Scale & 6 \\
Therapy Outcome Measures (TOMS) & 4 \\
Barthel Index & 6 \\
FIM-FAM & 3 \\
Dynamic Gait Index & 3 \\
Get Up and Go & 2 \\
Other Outcome Measures & 5 \\
Total & 65 \\
\hline
\end{tabular}

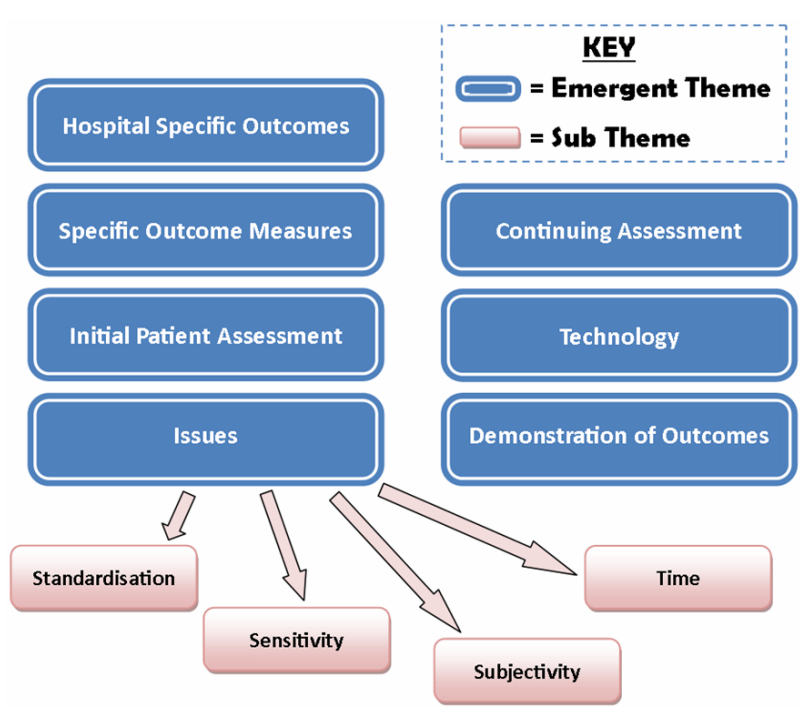

Figure 1. Graphical representation of emergent themes and subthemes used to analyze interviews.

exceptional recovery. The patient described how they had been unable to even walk at first, but now their upper and lower limbs were all highly functional, albeit some minor musculoskeletal problems due to the stroke. The only factors which had stopped the patient making a fully-fledged recovery were, in fact, problems prior to the stroke; an injured shoulder which affected Range of Motion (ROM), a previously broken finger (also affected ROM) and, finally, balance difficulties which were heightened by the stroke.

The therapist detailed before the session that this was not an initial assessment, and as this had been performed previously there would not be a great amount of assessment taking place. However, once arriving in the session it became very clear that the therapist was constantly assessing the patient during every movement and between each exercise. It should be noted how integrated assessment is within rehabilitation and it is not always conducted as a separate process.

The patient began the session supine. Whilst in this position the therapist began to evaluate their ROM. The therapist was mainly focused on the abduction/adduction of the left shoulder joint and the extension/flexion of the elbow joint. Supination and pronation were constantly happening during this time, however, the patient was not aware of this (the therapist later explained that they pay particular attention to this supination/pronation at the elbow joint as this is a highly problematic yet very common problem in individuals with stroke).

It became very clear as the session continued that the therapist was constantly checking the individual's ROM in various joints. What was particularly striking was how ad-hoc this procedure was. The therapist checked the patient's ROM and then seemingly from memory decided whether the patient had progressed. Very rarely would the therapist write or check notes to see the patient's progress. Even when notes were taken it became very clear how qualitative and ad-hoc the whole procedure was, with almost random notes being taken.

Throughout the session, during all activities and assessments, the therapist would be constantly supporting various body parts of the patient (e.g. supporting the elbow joint whilst checking flexion, extension and deviation of the wrist joint). This was due to the patients being unable to hold their limbs in certain positions, which would allow the therapist to assess other joints. This was particularly apparent when the patient was asked to hold their arm in the air and it simply dropped down at a slow speed, with the patient completely unaware of the phenomenon.

The therapist then proceeded to conduct a task whereby they held their arm high in the air and asked the patient to touch their arm ten times. The patient was able to do this but they were clearly having difficulties. What was quite noticeable at this point was the change in the patient's behaviour as they seemingly became emotionally agitated at the fact that they struggled to carry out the task. Motivation was a massive factor at this point as the patient may have lost the will to continue.

The next test which was conducted was a sensitivity assessment. The patient was asked to rate their sense of feeling on a scale of one to ten when the therapist touched firstly the front of their hand, and latterly the back of their hand. The patient commented at this moment that they had much more feeling on the palm of their hand than on the back. They also commented that they were unable to place their hand in hot water. A test of sensitivity then continued whereby the patient was asked to close their eyes whilst the therapist touched the end of the patient's fingers with a pen. The patient was then asked to state which digit had been touched. This sense of touch is very 
important in therapy.

The therapist then moved on to testing the patient's grip. The patient spoke about how they experienced pain whilst grasping objects and this prevented them from practising this. At this point, the patient also mentioned that their vision was poor and they had recently had a fall because they were unable to see a chair clearly and attempted to sit down too far in front of it. The therapist now carried out a grip test, asking the patient to grasp and release a cup ten times. Whilst they were performing this exercise they were asked to keep their elbow firmly on the table in order to support it (as done previously by the therapist). Grip tests were also affected by mild spasticity in the hand. Another problem which occurred was the patient moving their shoulder forwards in order to "propel” their arm forwards to avoid using muscle groups. The therapist said later that this is a very common compensatory problem amongst stroke patients.

The patient commented that they receive a lot of motivation from their caregiver and the therapist later commented that there is a far greater improvement in patients who have a caregiver. The therapist was providing constant "hints" to the patient throughout the session (e.g. put weight on your left leg, keep your back straight). The patient did state that they required constant "hints" as they struggled to concentrate on two things at once.

\subsection{Non-Participant Observation of Occupational Therapist with Patient 2}

Patient 2 arrived for their therapy session in an awkward manner due to experiencing a large amount of back pain, which the therapist believed to be not directly related to the stroke. This patient also appeared to have made a very good recovery from stroke, although it was difficult to ascertain a detailed level of information due to cognitive difficulties of the patient coupled with stress from the pain in their back.

The first point that the therapist commented on was the patient's ROM (an apparent improvement of around 40 degrees abduction, with extension at the elbow up to around 80 degrees). The therapist stated that this was a massive improvement. However this was from the therapist's memory. Something of particular note was that the therapist called the patient by the wrong name several times, and although possibly finicky, if this is the case then is it possible that they may be getting the patient's ROM mixed up with another patient? Quantitative data analysis with stored progress data may be a way to alleviate this issue.

The OT now proceeded to conduct a task whereby they placed their hand in the air and asked the patient to touch their hand ten times. The patient carried out this task quite efficiently, although appeared to be in a lot of difficulty with back pain.
The therapist then asked the patient to pour some water into a cup. The patient completed this but again with back pain. However, it was noticeable that some movements were not correct when pouring the water (e.g. pronation of the forearm). Their elbow was supported by the therapist during the task.

The therapist then asked the patient to carry out some simple tasks involving folding and rolling a towel. The patient completed this but with some incorrect movements. The therapist was constantly telling the patient minor details that they needed to correct. They were asked to continue this at home, as well as practicing picking up and releasing various sized objects.

The therapist now proceeded with strength tests involving a dynamometer and a pinch gauge. This was the first point at which quantitative data was taken and compared with previous sessions.

The therapist completed the session by asking the patient to close their eyes, whilst the therapist touched each of the patient's fingers with a pen, and asked the patient to state which digit had been touched. The patient struggled with this and the therapist stated that it was something that would need to be addressed at a later point.

This session was eventually ended prematurely due to the patient's back problems (which were not directly linked to the stroke) with the therapist recommending that the patient seek the advice of a General Practitioner (GP).

\subsection{Non-Participant Observation of Physiotherapist with Patient 3}

This first observation was a small part of an initial assessment session with a new patient that had just began treatment at the rehabilitation hospital. The session was kept very short due to the patient having speech and hearing impairments (no speech or hearing) before their stroke. I was informed that a sign language expert was due to arrive at the hospital to assist the patient in their rehabilitation; but as this had not yet happened only minimal assessment was performed. There were two PTs present for this session (one therapist was an assistant to the second therapist, and had entered the room purely to assist in certain areas).

The PTs were very "hands-on" throughout the session and mainly focused on checking the patient's ROM and their muscular strength, often through resistance tests.

The session began by asking the patient to move their feet. First of all, plantar and dorsi flexion at the ankle were assessed, however, problems were already arising in communicating to the patient what exactly to move. The patient managed to perform this task, however it was obvious that even though the patient was able to flex their right foot, it was still quite a slow movement and was inhibited by the stroke.

The PT then checked the patient's flexion and exten- 
sion at their knee joint. This was the first instance at which resistance was used, pushing lightly on the patient's ankle to see if they could still extend their leg. The patient struggled to completely extend their leg and needed some help with completing this task.

The patient was then asked to perform knee raises; again, resistance was applied but the patient managed to finish this exercise quite easily.

The PT then proceeded to assess the patient's upper limbs. First of all, flexion and extension at the shoulder joint were tested. The patient was asked to fully flex their arm at the right shoulder and place their hand behind their back. The patient was unable to achieve this without assistance and actually experienced pain around their right scapula, so the PT ceased this task.

The patient was then asked to extend the fingers on their right hand. The patient kept "flicking" their right hand, becoming increasingly agitated at their inability to control their fingers. The PT moved on to another test promptly. This is an area that would have to be addressed during therapy, but the PT proceeded quickly as there was no need to agitate the patient during their initial assessment.

The patient was then asked to flex their arm at the elbow joint. The patient needed assistance in performing this task with their right arm. At this time the PT also checked for abduction in the patient's right arm, however, with pain still present in the right shoulder, this task was ceased.

Both PTs then proceeded to help the patient stand (the patient had originally been in a wheelchair). The patient seemed to struggle with this and it seemed to take a lot of effort by the PTs. The patient also required a support strap placed around their back. The PTs used this strap to support the patient while standing. One PT began checking for hyperextension at the knee joint whilst the patient was standing.

The PTs then moved the patient to the bed, sitting down, so they could perform further assessment. A length of time was now used where the patient was trying to tell the PTs something (hindered by speech difficulties). The patient used a board with letters on to try to spell out what they wanted to say. They also tried writing sentences down on a piece of paper. The patient appeared anxious during this period and the PT constantly gave motivational support and this appeared to be an important part of the rehabilitation process. The patient managed to state that they used to walk and drive and that they have only been walking a small amount since the stroke. The PT then told the patient that they would set goals that targeted allowing the patient to walk again.

The patient was then asked to move to the supine position. They were asked to plantar flex their feet and flex their knees whilst carrying out extension at the hip joint.
The patient's right knee was not stable during this task (as a result of the stroke) and they were asked to concentrate on keeping their knee straight. The patient was then stood up again and the PTs helped the patient walk out of the room. The patient seemed very confident in performing this activity.

\subsection{Initial Patient Assessment}

All therapists that were interviewed commented on an initial assessment that patients underwent once they arrived at the ward, with most therapists making comments such as "I do my initial assessment right at the beginning” (PT4). The method as to which the patients are assessed can vary. Some therapists said that they used standardized outcome measures: "using the Berg Balance when I first see them" (PT3). However, a lot of therapists spoke about how they used an assessment form that was created within the hospital itself: "we have an initial assessment” (OT3).

\subsection{Continuing Assessment}

Therapists described how they do not use standard outcome measures often, but state: "realistically, every time I see a patient I reassess” (PT3). PT3 also stated that there was no set time for formal assessments of the patient, excluding initial assessment and sometimes "on discharge home", whilst PT1 stated: "they [patients] are not reviewed on a regular basis". This was a recurring response (mentioned, unprompted, on eleven separate occasions), with one therapist stating: "There is no timeframe where patients need to be assessed after $X$ amount of time, it is just an individual clinicians decision” (OT5). This is even though a general consensus existed concerning the importance of continuous assessment: "I think that the assessment needs to happen continuously... whether the resources are there to support that is a completely and entirely different issue" (OT1). It was also described that what is assessed changes from session to session, and patient to patient: "I may reassess everything in a couple of weeks or I may not reassess anything until discharge" (PT4).

\subsection{Hospital Specific Outcomes}

In close contrast with initial patient assessment, some therapists described outcome measures that had been created within their own hospital, and only existed in that hospital: "we just use our own assessment form" (OT4); "we have our own paperwork for upper limb, washing and dressing" (OT2).

One interviewee discussed this by saying: "The way we get around that here, is on our assessment form. We have a description of what each grade is so only those grades are used. So, whilst it's not an ideal score, it's the 
only one we've got in the absence of expensive equipment which we don't have access to" (PT1).

\subsection{Issues}

Time-Eight interviewees described how time constraints affected their ability to perform adequate assessment using outcome measures: "Something that stops us using them a lot is the time factor" (OT4); "I'd say time constraints [are a weakness], with the proportion of patients to staff" (PT4); "We wouldn't have time to treat patients and use the scales" (OT4).

Subjectivity-A re-occurring theme throughout the interviews was that of subjectivity and inter-rater reliability of outcome measures. Therapists described how outcome measures are "... quite subjective really, and the patient score isn't always correct" (OT2), and that they "are quite open to interpretation" (PT1). This theme was mentioned on 21 separate occasions during the course of the interviews.

Multiple therapists discussed inter-rater reliability of outcome measures and how "What someone's 4/5 might be, somebody else might think is a 3/5" (OT5). Another therapist stated that due to these inconsistencies: "People do not necessarily use them [outcome measures] appropriately. The guidance information on how you carry them out is not always clear" (PT1), whilst another professional added: "Junior therapists, I would actually say they struggle with knowing what to do" (OT1).

Standardization-A strong emerging theme in these interviews is the lack of standardization: "There isn't a consistent service out there" (PT2); "I imagine everywhere assesses slightly differently” (OT5).

Therapists described differences in outcome measures between hospitals: "Some places are not as good at using standardized outcome measures as others” (PT1); “That's just what we use in our hospital [outcome measures], and it will definitely be different to what everybody uses in every other hospital" (OT4). It was also described that there was a difference between assessments in departments: "Different departments tend to be towards one side or the other, and that can cause discrepancies between the units... it is not standardized [outcome measures] across this hospital site at all" (PT1). One therapist described how they felt disbelief in the system: "There's probably a lack of training at the university level” (OT1), describing how this leads to discrepancies between outcome measures. This was described more comprehendsively with regards to outcome measures: "We use a range of outcome measures, but we wouldn't necessarily use the same ones with each person" (PT5); "We pick and choose which ones [outcome measures] we use with each patient, rather than using the same for everybody" (PT4).

Another problem area, in addition to this, was that of regulation between specialties: "We probably need to have a little bit more standardization inter-disciplinary wise" (OT1). One therapist stated, when talking about results of outcome measures: "I am not actually sure that anybody does anything with the data” (OT3), whilst PT1 said "We are not meeting the standards set out in the stroke strategy".

Sensitivity-Three of the therapists that were interviewed described how outcome measures “don't always pick up all of the problems the patient may have" (PT5) and "Improvement in our patients is often quite a small thing to measure and we can see that they have made some improvements but it doesn't show on the scale. They are not sensitive enough for them" (OT4). Other concerns that were raised about scales related to "cognition [and] communication" (PT1) issues, which made it difficult to administer outcome measures. It was also discussed that "all [outcome measures] have got some kind of flaw to them" (PT1) and "a lot of them have ceiling and floor effects" (PT4).

\subsection{Specific Outcome Measures}

Sixteen separate outcome measures were spoken about during the course of the interviews. Each test described has been used by a therapist within the hospital they are now employed by. Table 2 illustrates the number of different outcome measures and how many times they were mentioned during interviews (if only mentioned once, the test was placed in "other outcome measures").

Table 2 demonstrates a broad spectrum of outcome measures used across two NHS sites, illustrating the variability of outcome measures not only between hospitals and departments, but also intra-departmental, between the therapists themselves.

\subsection{Demonstration of Outcomes}

A theme that emerged was that it would be desirable to have a method of demonstrating assessment outcomes to patients, staff and families, "so someone can see where they are" (PT3). One therapist stated: "I think it would probably be helpful for both therapist and patient if there was a way of collating all of the measures that are used in a way that... can be turned into graphs, something quite visual” (PT1).

The same therapist discussed a lack of methods for representing the progress of patients: "Possibly having some kind of visual aid might help the therapist" (PT1). One professional claimed that if there was some kind of visual feedback then "It's a really good way of assessing people as you can go back and look at it and it is a lot easier to compare” (PT5). One therapist also stated that "you could use that kind of information [visual assessment data] to give to commissioners, for instance, to 
prove that the system is cost effective" (PT1).

\subsection{Technology}

Therapists were asked about how they felt technology could contribute towards the assessment of stroke patients for rehabilitation. Many discussed technologies that are already in use such as "biometrics" (OT1), and, stated that a tool that "anybody can go and use" (OT1) which accurately assesses an individual would be useful, as patients "quite like having a number" (PT1) which describes rehabilitative progress. It was also mentioned that remote assessment of patients from within the home using computers and the internet could be useful: "Thinking about being in an office and being able to see somebody in their home would be great" (PT3). They described how using technology to look "at something like that in terms of exercise programs... using the computer or television" (OT1). Another therapist stated that "Maybe if there was something you can leave with the patient, to help them practice movement” (PT4).

However, one therapist did state that "I think hands-on assessments are a better idea, than using technology to do that for you" (OT2), indicating how boundaries do exist such as user acceptance of technology.

Therapists also discussed how these boundaries exist with patients: "because of cognitive difficulties, some patients don't grasp the technology" (PT4). However, some therapists felt that attitudes towards technology are changing: "I think a lot of patients; even the elderly patients... are computer literate and have a computer in the house" (PT3). "You're getting... younger patients with technology knowledge" (OT2), explaining an increase in technology acceptance.

\subsection{Coping with Stroke}

All interviewees raised the issue of the effects that stroke has on individuals. Some described how patients suffer with movement issues, and assessment can require movement analysis, as well as looking at areas such as "kitchen assessments [and] mobility transfer" (OT2). It was also noted that assessment for strokes will always be difficult as "Their recovery can be so different from one to another" (OT2).

\section{DISCUSSION}

Qualitative thematic analysis is a recognized method for evaluating service delivery, professional practice and patient experience and can identify areas of concern within the field of healthcare. In fact, Buck et al. [9] state that more in-depth interviews and qualitative research are required within the area of assessment for stroke patient rehabilitation to identify the needs of this field.

In recent times there has been a move to increase the standardization of care using evidence based practice. With budget cuts now being enforced, the NHS is obligatorily streamlining systems and asking for "More with the same, not more of the same" [14]. With questions being raised about the rehabilitation of stroke patients [5-8], the $£ 8.9$ billion it costs to the UK economy per year [15], and the doubts over assessment methods being raised [912], this paper helps to illustrate an area of concern that could be improved and standardized in order to create "more with the same".

This study identified that outcome measures are currently not being used in a standardized way within NHS hospitals, and due to this, staff struggle to establish feedback methods that are so essential to rehabilitation, as outlined in the National Clinical Guidelines for Stroke [4]. Therapists will assess a patient once they arrive in a rehabilitative setting, and will sometimes assess on discharge. However, it was apparent that continuing formalized assessment was not being implemented. This means that no feedback can be provided to the patient, their families, healthcare commissioners or indeed the therapists themselves.

Therapists expressed distrust in outcome measures and were often performing more informal assessments each time a patient entered therapy. However, concerns were shown with these methods of assessment, particularly due to time taken (which distracted from therapy time), subjectivity of assessments and standardization across departments and hospitals.

Standardization across hospitals is of importance and it is shown here that not only are hospitals using different outcome measures to other hospitals (with sixteen separate scales mentioned by only ten therapists), but they are creating their own assessment methods.

\section{LIMITATIONS}

The authors of this paper are aware that this study only interviewed therapists from one region of the UK. Further research is required to ascertain how applicable these results are to other NHS areas.

However, many of the staff we interviewed had worked at different hospitals around the UK, and even outside of it, making it less likely that these findings are only applicable to one area of the country.

\section{CONCLUSION}

All therapists interviewed and observed in this study were clear that they believed that change is required within this field, whether through emphasis on the development of assessment technologies, or through more standardized use of outcome measures, particularly in a more quantifiable manner.

However this is achieved, the field of stroke patient assessment for musculoskeletal rehabilitation is an area 
that should be viewed as an area of concern and a field that could be streamlined to adhere to the UK Department of Health's strategy: "more with the same, not more of the same".

\section{ACKNOWLEDGEMENTS}

The research team would like to thank the Engineering and Physical Sciences Research Council (EPSRC) and the Economic and Social Research Council (ESRC) for funding this study. We would also like to particularly thank all participants at the two NHS sites who agreed to participate in this study.

\section{REFERENCES}

[1] Mackay, J. and Mensah, G. (2004) The atlas of heart disease and stroke. World Health Organization, Geneva.

[2] O’Donnell, M.J., et al. (2010) Risk factors for ischaemic and intracerebral haemorrhagic stroke in 22 countries (the INTERSTROKE study): A case-control study. The Lancet, 376 112-123. doi:10.1016/S0140-6736(10)60834-3

[3] National Institute for Health and Clinical Excellence (2008) Stroke: National clinical guideline for diagnosis and initial management of of acute stroke and transient ischaemic attack (TIA). NICE, London.

[4] Intercollegiate Stroke Working Party (2012) National clinical guidelines for stroke. Royal College of Physicians, London.

[5] Young, J. and Forster, A. (2007) Review of stroke rehabilitation. British Medical Journal, 334, 86. doi:10.1136/bmj.39059.456794.68

[6] Tyson, S. and Turner, G. (1999) The process of stroke re- habilitation: What happens and why. Clinical Rehabilitation, 13, 322. doi:10.1191/026921599674794965

[7] Smith, J., Forster, A. and Young, J. (2008) Service needs and delivery following stroke: Evidence based review. University of Leeds and Commission for Rural Communities. www.ruralcommunities.gov.uk/files/stroke\%20Report\%20 formated

[8] Kwan, J. (2007) Care pathways for acute stroke care and stroke rehabilitation: From theory to evidence. Journal of Clinical Neuroscience, 14, 189-200. doi:10.1016/j.jocn.2006.01.026

[9] Buck, D., et al. (2000) Evaluation of measures used to assess quality of life after stroke. Stroke, 31, 2004-2010. doi:10.1161/01.STR.31.8.2004

[10] de Haan, R., et al. (1993) Measuring quality of life in stroke. Stroke, 24, 320-327. doi:10.1161/01.STR.24.2.320

[11] Jette, D.U., et al. (2009) Use of standardized outcome measures in physical therapist practice: Perceptions and applications. Physical Therapy, 89, 125-135. doi:10.2522/ptj.20080234

[12] Skinner, A. and Turner-Stokes, L. (2006) The use of standardized outcome measures in rehabilitation centres in the UK. Clinical Rehabilitation, 20, 609-615. doi:10.1191/0269215506cr981oa

[13] Bryman, A. (2004) Social research methods. 2nd Edition, Oxford University Press, New York, 592.

[14] Appleby, J., et al. (2010) Improving NHS productivityMore with the same, not more of the same. The Kings Fund, London.

[15] Saka, Ö., McGuire, A. and Wolfe, C. (2009) Cost of stroke in the United Kingdom. Age and Ageing, 38, 27-32. doi:10.1093/ageing/afn281 